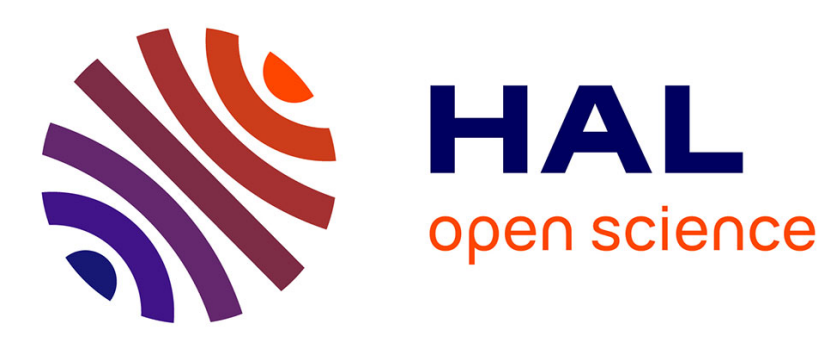

\title{
Force and Stiffness of Passive Magnetic Bearings Using Permanent Magnets. Part 1: Axial Magnetization
}

Romain Ravaud, Guy Lemarquand, Valérie Lemarquand

\section{To cite this version:}

Romain Ravaud, Guy Lemarquand, Valérie Lemarquand. Force and Stiffness of Passive Magnetic Bearings Using Permanent Magnets. Part 1: Axial Magnetization. IEEE Transactions on Magnetics, 2009, 45 (7), pp.2996. 10.1109/TMAG.2009.2016088 . hal-00413107

\section{HAL Id: hal-00413107 https://hal.science/hal-00413107}

Submitted on 3 Sep 2009

HAL is a multi-disciplinary open access archive for the deposit and dissemination of scientific research documents, whether they are published or not. The documents may come from teaching and research institutions in France or abroad, or from public or private research centers.
L'archive ouverte pluridisciplinaire HAL, est destinée au dépôt et à la diffusion de documents scientifiques de niveau recherche, publiés ou non, émanant des établissements d'enseignement et de recherche français ou étrangers, des laboratoires publics ou privés. 


\title{
Force and Stiffness of Passive Magnetic
} Bearings Using Permanent Magnets.

\section{Part 1: Axial Magnetization}

\author{
R. Ravaud, G. Lemarquand Senior IEEE and V. Lemarquand
}

\begin{abstract}
This paper deals with the calculation of the force and the stiffness between two ring permanent magnets whose polarization is axial. Such a configuration corresponds to a passive magnetic bearing. All the calculations are determined by using the Coulombian model. This paper also discusses the optimal ring dimensions in order to have a great force or a great stiffness between the rings. Such properties are commonly searched in passive magnetic bearings and we propose a three-dimensional method allowing to optimize these parameters. Furthermore, an important result is established in this paper: the exact relative position of the rings for which the force is the strongest depends on the air gap dimension. As the expressions presented in this paper give this exact relative position, manufacturers can easily optimize their passive magnetic bearings. It is noted that this result is new because the curvature effect is taken into account in this paper. Furthermore, such semi-analytical expressions are more precise than the numerical evaluation of the magnetic forces obtained with the finite element method. In addition, semi-analytical expressions have a low computational cost whereas the finite element method has a high one. Thereby, as shown in this paper, such calculations allow an easy optimization of quadripolar lenses or devices using permanent magnets.
\end{abstract}

\section{Index Terms}

Magnetic forces, analytical calculation, ring permanent magnet, magnetic bearing

manuscript received september 26,2008, first revision october 15, second revision january 20,2009.

The authors are with the Laboratoire d'Acoustique de l'Universite du Maine UMR CNRS 6613, Avenue Olivier Messiaen, 72085 Le Mans Cedex 9, France, corresponding author guy.lemarquand@iee..org 


\section{INTRODUCTION}

Passive magnetic bearings are commonly manufactured with permanent magnets axially or radially magnetized. The quality of such devices depend on the magnet material, its magnetic polarization and the dimensions of all the magnets used. The first studies concerning passive magnetic bearings with permanent magnets have been done by Yonnet [1][2]. Such passive magnetic bearings used only permanent magnets radially or axially magnetized. Permanent magnets are commonly used in many electrical devices and engineering applications. Indeed, most engineering applications need several ring permanent magnets and the determination of the magnetic force between them is thus required. It is to be noted that several way of obtaining magnetic forces between rings are possible. Authors generally use either numerical means or $2 \mathrm{D}$ analytical calculations for the determination of the magnetic field or of the magnetic forces created between ring permanent magnets [3]-[5]. Even if numerical means are very interesting for evaluating the mechanical properties of structures using ring permanent magnets, we think that analytical or semianalytical expressions are more useful because they have a low computational cost. Indeed, the major problem of a fully numerical approach lies in the fact that it has a high computational cost. Consequently, alternative solutions are thus required. Some authors have proposed semi-analytical expressions of the magnetic field created by ring permanent magnets [6]-[11]. However, according to our knowledge, no papers have been written concerning the three-dimensional calculation of the magnetic force or the magnetic stiffness between ring permanent magnets. In any case, some approaches allow to have a very low computational cost [12]-[20] for the determination of the magnetic forces exerted between ring permanent magnets. It is noted that it exists another way of calculating the forces between ring permanent magnets [21]-[24]. The 2D analytical approach allows an easy optimization of the ring dimensions but this approach is not very precise [25]-[28] if the ring radius is small. When the ring permanent magnets are axially magnetized, the magnetic field components can be determined at any point of the space [29]. Such an approach is appropriate because the algorithms used to calculate elliptic integrals are both very robust and fast (less than $0,2 \mathrm{~s}$ to determine the magnetic components of the field created by ring permanent magnets whose polarization is axial). Another interesting papers show that the calculation of the force between thin coaxial circular coils in air is possible by using special functions [30].

First, this paper presents useful semi-analytical expressions of the force and the stiffness exerted between two ring permanent magnets whose polarization is axial. Then, this paper deals with the optimization of the ring dimensions in order to have either a great force or a great stiffness between the rings. We show that this way of determining these mechanical properties allows us to optimize precisely the ring dimensions. 
All the expressions determined in this paper are available online [31].

\section{CAlculation of the AXIAL FORCE BETWEen THE TWO RING PERMANENT MAGNETS}

This section presents a semi-analytical calculation of the force exerted between two ring permanent magnets whose polarization is axial. It is noted that the two ring permanent magnets are radially centered. The interest of using ring permanent magnets axially magnetized lies in the fact that they are very simple to realize.

\section{A. Notation and geometry}

The geometry considered is shown in Fig 1. A two dimensional representation of the passive magnetic bearing is shown in Fig 2. We can say that such a configuration corresponds to an axial bearing. The outer radius of the outer ring is $r_{\text {out }}$ and the inner one is $r_{\text {in }}$. The outer ring height is $h$. The outer radius of the inner ring is $r_{\text {out } 2}$ and the inner one is $r_{i n 2}$. The ring inner height is $z_{b}-z_{a}$. It is to be noted that the Coulombian model of a permanent magnet is used. As a consequence, each ring permanent magnet is represented by two charged planes located on the upper and lower faces of each ring. For the outer ring, the upper face is charged with the magnetic pole surface density $+\sigma^{*}$ and the lower face is charged with the magnetic pole surface density $-\sigma^{*}$. For the inner ring, the upper face is charged with the magnetic pole surface density $-\sigma^{*}$ and the lower face is charged with the magnetic pole surface density $+\sigma^{*}$. Moreover, it is noted that all the illustrative calculations are done with $\sigma^{*}=\vec{J} \cdot \vec{n}=1 \mathrm{~T}$ where $\vec{J}$ is the magnetic polarization vector and $\vec{n}$ is the unit normal vector which is directed towards 0 .

\section{B. Semi-analytical expression of the magnetic force}

The axial passive magnetic bearing studied has two ring permanent magnets which are supposed to be radially centered. Consequently, there is only the axial component of the magnetic force which is exerted between the two rings. We call it the axial force $F_{z}$. This axial force can be determined by integrating the magnetic field created by the outer ring on the charge contributions of the inner one [1]. By denoting $H_{z}$, the axial component of the magnetic field produced by the outer ring permanent magnet, the axial force $F_{z}$ can be written as follows:

$$
F_{z}=-\iint_{\left(S_{\text {up }}\right)} H_{z} \sigma_{2}^{*} d \tilde{S}+\iint_{\left(S_{\text {down }}\right)} H_{z} \sigma_{2}^{*} d \tilde{S}
$$




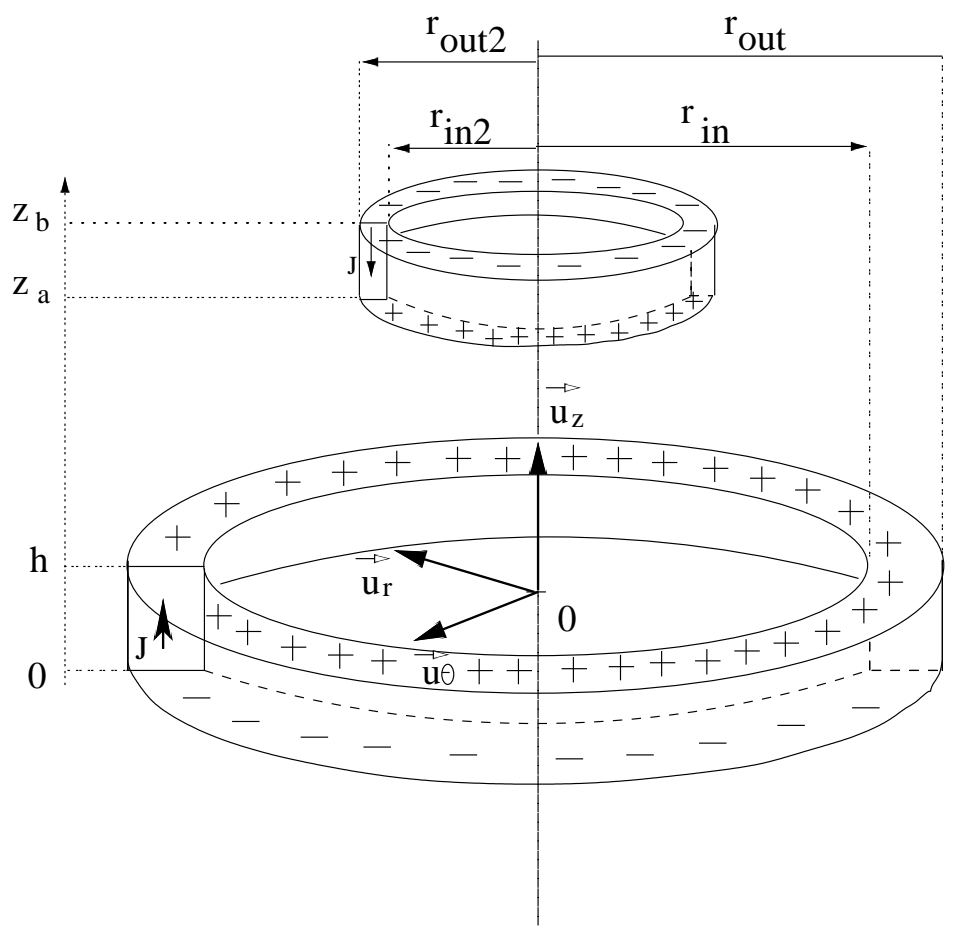

Fig. 1. Representation of two ring permanent magnets axially magnetized. The inner radius of the outer ring is denoted $r_{i n}$, the outer one is $r_{\text {out }}$, its height is $h$. The inner radius of the inner ring is denoted $r_{i n 2}$, the outer one is $r_{\text {out } 2}$, its height is $z_{b}-z_{a}$.

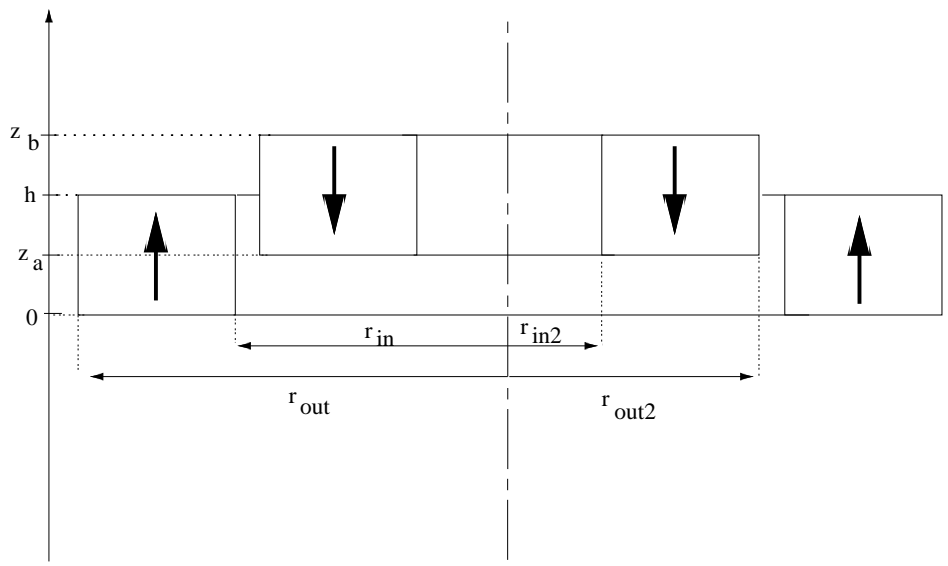

Fig. 2. Axial magnetic bearing using magnets radially magnetized for mutual attraction. The inner radius of the outer ring is denoted $r_{i n}$, the outer one is $r_{\text {out }}$, its height is $h$. The inner radius of the inner ring is denoted $r_{\text {in } 2}$, the outer one is $r_{\text {out } 2}$, its height is $z_{b}-z_{a}$. 
where $S_{u p}$ is the upper face of the inner ring permanent magnet and $S_{\text {down }}$ is the lower face of the inner ring permanent magnet. We deduct that this axial force can be expressed as follows:

$$
\begin{aligned}
F_{z}= & -\int_{\theta_{1}=0}^{2 \pi} \int_{\theta_{2}=0}^{2 \pi} \int_{r_{1}=r_{\text {in }}}^{r_{\text {out }}} \int_{r_{2}=r_{\text {in } 2}}^{r_{\text {out } 2}} z_{a} a\left(z_{a}\right) r_{1} r_{2} d r_{1} d r_{2} d \theta_{1} d \theta_{2} \\
& -\int_{\theta_{1}=0}^{2 \pi} \int_{\theta_{2}=0}^{2 \pi} \int_{r_{1}=r_{\text {in }}}^{r_{\text {out }}} \int_{r_{2}=r_{\text {in } 2}}^{r_{\text {out } 2}}\left(z_{a}+b-h\right) a\left(z_{a}+b-h\right) r_{1} r_{2} d r_{1} d r_{2} d \theta_{1} d \theta_{2} \\
& +\int_{\theta_{1}=0}^{2 \pi} \int_{\theta_{2}=0}^{2 \pi} \int_{r_{1}=r_{\text {in }}}^{r_{\text {out }}} \int_{r_{2}=r_{\text {in } 2}}^{r_{\text {out } 2}}\left(z_{a}+b\right) a\left(z_{a}+b\right) r_{1} r_{2} d r_{1} d r_{2} d \theta_{1} d \theta_{2} \\
& +\int_{\theta_{1}=0}^{2 \pi} \int_{\theta_{2}=0}^{2 \pi} \int_{r_{1}=r_{\text {in }}}^{r_{\text {out }}} \int_{r_{2}=r_{\text {in } 2}}^{r_{\text {out } 2}}\left(z_{a}-h\right) a\left(z_{a}-h\right) r_{1} r_{2} d r_{1} d r_{2} d \theta_{1} d \theta_{2}
\end{aligned}
$$

with

$$
a(\xi)=\frac{\sigma_{1}^{*} \sigma_{2}^{*}}{4 \pi \mu_{0}} \frac{1}{\left(r_{1}^{2}+r_{2}^{2}-2 r_{1} r_{2} \cos (\theta)+\xi^{2}\right)^{\frac{3}{2}}}
$$

where $\mu_{0}$ is the permeability of the vacuum, $b=z_{b}-z_{a}, \sigma_{1}^{*}$ is the magnetic pole surface density owing to the outer ring permanent magnet and $\sigma_{2}^{*}$ is the magnetic pole surface density owing to the inner ring permanent magnet. After three analytical integrations according to $r_{1}, r_{2}$ and $\theta_{2}$, we obtain a semianalytical expression of the axial force using only one numerical integration. As the expression obtained own only one numerical integration, its calculation is very fast.

$$
\begin{gathered}
F_{z}=\frac{\sigma_{1} \sigma_{2}}{2 \mu_{0}} \int_{\theta=0}^{2 \pi}(A(0)+A(h-b)-A(-b)-A(h)) d \theta \\
A(x)=f\left(x, z_{a}, r_{\text {out }}, \theta, r_{\text {in } 2}, r_{\text {out } 2}\right)-f\left(x, z_{a}, r_{\text {in }}, \theta, r_{\text {in } 2}, r_{\text {out } 2}\right) \\
f\left(x, z_{a}, y, \theta, r_{\text {in } 2}, r_{\text {out } 2}\right)=X\left(\left(z_{a}-x\right)^{2}, y^{2}, y \cos (\theta), \cos (2 \theta), r_{\text {in } 2}, r_{\text {out } 2}\right)
\end{gathered}
$$

$$
\begin{gathered}
X\left(a, b, d, g, r_{2}\right)=-\frac{\sqrt{a}}{2(1+g)^{2}}\left(4(1+g) \sqrt{a+b+r_{2}\left(-2 d+r_{2}\right)}+\frac{\alpha_{1} \log \left[\beta_{1}\right]}{\sqrt{\xi_{1}}}-\frac{\alpha_{2} \log \left[\beta_{2}\right]}{\sqrt{\xi_{2}}}\right) \\
\alpha_{1}=(-1)^{\frac{3}{4}} \sqrt{2 a}(\sqrt{2 a}(-1+g) \sqrt{1+g}+2 i d(1+g)) \\
\alpha_{2}=(-1)^{\frac{1}{4}} \sqrt{2 a}(\sqrt{2 a}(-1+g) \sqrt{1+g}-2 i d(1+g))
\end{gathered}
$$




$$
\begin{gathered}
\beta_{1}=\frac{-2\left(\beta_{11}+\beta_{12}\right)(1+g)^{2}}{\xi_{1} \sqrt{a}(\sqrt{2 a}(-1+g) \sqrt{1+g}+2 i d(1+g))\left(\sqrt{2 a} \sqrt{1+g}-i(1+g) r_{2}\right)} \\
\beta_{11}=(1+i)(b+b g+a(1+g))+2(-1)^{\frac{3}{4}} \sqrt{a} \sqrt{1+g}\left(d-r_{2}\right) \\
\beta_{12}=-(1+i)\left(d r_{2}+d g r_{2}\right)+i \sqrt{2} \sqrt{1+g} \xi_{1} \sqrt{a+b-2 d r_{2}+r_{2}^{2}} \\
\beta_{2}=\frac{2(1+g)^{2}\left(\beta_{21}+\beta_{22}\right)}{\xi_{2} \sqrt{a}(\sqrt{2 a}(-1+g) \sqrt{1+g}-2 i d(1+g))\left(\sqrt{2 a} \sqrt{1+g}+i(1+g) r_{2}\right)} \\
\xi_{21}=-(1-i)(b+b g+a(1+g))+2(-1)^{\frac{1}{4}} \sqrt{a} \sqrt{1+g}\left(d-r_{2}\right) \\
\beta_{22}=(1-i) d r_{2}(1+g)+i \sqrt{2} \sqrt{1+g} \xi_{2} \sqrt{a+b-2 d r_{2}}+r_{2}^{2} \\
\xi_{2}=\sqrt{i a(-1+g)+2 \sqrt{2 a} d \sqrt{1+g}+i b(1+g)}
\end{gathered}
$$

Figure 3 represents the axial force exerted between two ring permanent magnets versus the axial displacement of the inner ring permanent magnet for the following dimensions: $r_{\text {in }}=0.025 \mathrm{~m}, r_{\text {out }}=$ $0.028 \mathrm{~m}, r_{\text {in } 2}=0.0219 \mathrm{~m}, r_{\text {out } 2}=0.0249 \mathrm{~m}, J=1 \mathrm{~T}, h=0.003 \mathrm{~m}, z_{b}-z_{a}=0.003 \mathrm{~m}$.

\section{Determination of the stiffness between two ring permanent magnets whose polarization is axial}

This section presents a semi-analytical calculation of the magnetic stiffness exerted between two ring permanent magnets whose polarization is axial. It is noted that the two ring permanent magnets are assumed to be radially centered. The axial stiffness $K_{z}$ can be determined as follows:

$$
K_{z}=-\frac{\partial}{\partial z_{a}} F_{z}
$$

where $F_{z}$ is the axial force (4) exerted between two ring permanent magnets. 


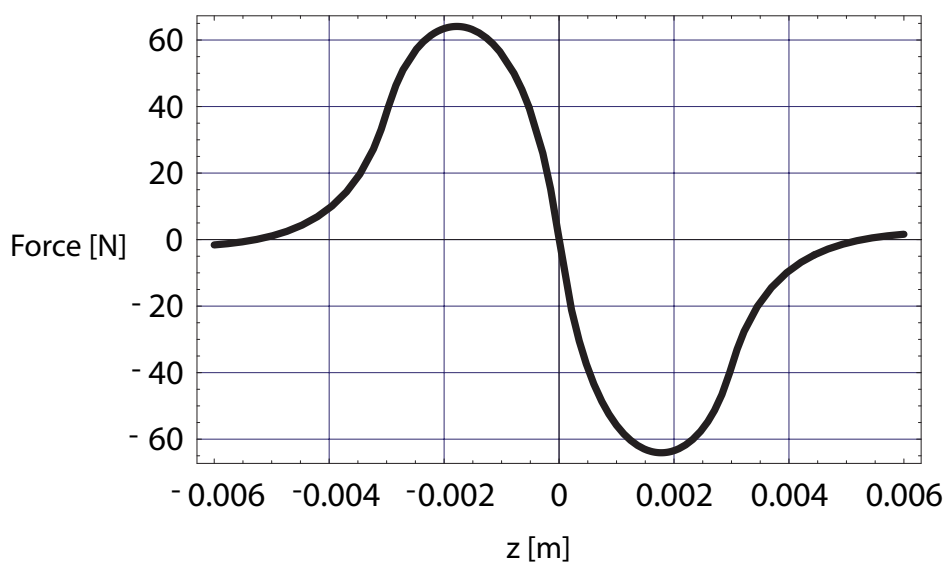

Fig. 3. Representation of the axial force exerted between two ring permanent magnets versus the axial displacement of the inner ring permanent magnet. The inner radius of the outer ring is denoted $r_{i n}$, the outer one is $r_{\text {out }}$, its height is $h$. The inner radius of the inner ring is denoted $r_{i n 2}$, the outer one is $r_{\text {out } 2}$, its height is $z_{b}-z_{a}$.

So, we obtain :

$$
\begin{aligned}
K_{z}= & +\frac{\partial}{\partial z_{a}} \int_{\theta_{1}=0}^{2 \pi} \int_{\theta_{2}=0}^{2 \pi} \int_{r_{1}=r_{\text {in }}}^{r_{\text {out }}} \int_{r_{2}=r_{\text {in } 2}}^{r_{\text {out } 2}} z_{a} a\left(z_{a}\right) r_{1} r_{2} d r_{1} d r_{2} d \theta_{1} d \theta_{2} \\
& +\frac{\partial}{\partial z_{a}} \int_{\theta_{1}=0}^{2 \pi} \int_{\theta_{2}=0}^{2 \pi} \int_{r_{1}=r_{\text {in }}}^{r_{\text {out }}} \int_{r_{2}=r_{\text {in } 2}}^{r_{\text {out } 2}}\left(z_{a}+b-h\right) a\left(z_{a}+b-h\right) r_{1} r_{2} d r_{1} d r_{2} d \theta_{1} d \theta_{2} \\
& -\frac{\partial}{\partial z_{a}} \int_{\theta_{1}=0}^{2 \pi} \int_{\theta_{2}=0}^{2 \pi} \int_{r_{1}=r_{\text {in }}}^{r_{\text {out }}} \int_{r_{2}=r_{\text {in } 2}}^{r_{\text {out } 2}}\left(z_{a}+b\right) a\left(z_{a}+b\right) r_{1} r_{2} d r_{1} d r_{2} d \theta_{1} d \theta_{2} \\
& -\frac{\partial}{\partial z_{a}} \int_{\theta_{1}=0}^{2 \pi} \int_{\theta_{2}=0}^{2 \pi} \int_{r_{1}=r_{\text {in }}}^{r_{\text {out }}} \int_{r_{2}=r_{\text {in } 2}}^{r_{\text {out } 2}}\left(z_{a}-h\right) a\left(z_{a}-h\right) r_{1} r_{2} d r_{1} d r_{2} d \theta_{1} d \theta_{2}
\end{aligned}
$$

where $a(\xi)$ is given by (3) and $b=z_{b}-z_{a}$.

After calculating the derivative with respect to $z_{a}$ and integrating according to $\theta_{2}$ and $r_{1}$, the axial stiffness can be expressed as follows:

$$
\begin{gathered}
K_{z}=\int_{\theta=0}^{2 \pi} \int_{r_{2}=r_{\text {in } 2}}^{r_{\text {out } 2}} \tilde{f} d r_{2} d \theta \\
\tilde{f}=f\left(h, z_{a}+b\right)-f\left(h, z_{a}\right)+f\left(0, z_{a}\right)-f\left(0, z_{a}+b\right)
\end{gathered}
$$




$$
\begin{aligned}
f(\alpha, \beta)= & -\frac{8 r_{2}(-\alpha+\beta)^{2}\left((\alpha-\beta)^{2}+r_{2}^{2}-r_{2} r_{\text {in }} \cos (\theta)\right)}{\sqrt{r_{2}^{2}+r_{\text {in }}^{2}+(\alpha-\beta)^{2}-2 r_{2} r_{\text {in }} \cos (\theta)}\left(2(\alpha-\beta)^{2}+r_{2}^{2}-r_{2}^{2} \cos (2 \theta)\right)^{2}} \\
& +\frac{8 r_{2}(-\alpha+\beta)^{2}\left((\alpha-\beta)^{2}+r_{2}^{2}-r_{2} r_{\text {out }} \cos (\theta)\right)}{\sqrt{r_{2}^{2}+r_{\text {out }}^{2}+(\alpha-\beta)^{2}-2 r_{2} r_{\text {out }} \cos (\theta)}\left(2(\alpha-\beta)^{2}+r_{2}^{2}-r_{2}^{2} \cos (2 \theta)\right)^{2}} \\
& +\frac{4 r_{2}(-\alpha+\beta)^{2}}{\sqrt{r_{2}^{2}+r_{\text {in }}^{2}+(\alpha-\beta)^{2}-2 r_{2} r_{\text {in }} \cos (\theta)}\left(2(\alpha-\beta)^{2}+r_{2}^{2}-r_{2}^{2} \cos (2 \theta)\right)^{2}} \\
& -\frac{2 r_{2}(-\alpha+\beta)^{2}}{\sqrt{r_{2}^{2}+r_{\text {out }}^{2}+(\alpha-\beta)^{2}-2 r_{2} r_{\text {out }} \cos (\theta)}\left(2(\alpha-\beta)^{2}+r_{2}^{2}-r_{2}^{2} \cos (2 \theta)\right)^{2}} \\
& -\frac{2 r_{2}(-\alpha+\beta)^{2}\left((\alpha-\beta)^{2}+r_{2}^{2}-r_{2} r_{\text {in }} \cos (\theta)\right)}{\left(r_{2}^{2}+r_{\text {in }}^{2}+(\alpha-\beta)^{2}-2 r_{2} r_{\text {in }} \cos (\theta)\right)^{\frac{3}{2}}\left(2(\alpha-\beta)^{2}+r_{2}^{2}-r_{2}^{2} \cos (2 \theta)\right)^{2}} \\
& +\frac{2 r_{2}(-\alpha+\beta)^{2}\left((\alpha-\beta)^{2}+r_{2}^{2}-r_{2} r_{\text {out }} \cos (\theta)\right)}{\left(r_{2}^{2}+r_{\text {out }}^{2}+(\alpha-\beta)^{2}-2 r_{2} r_{\text {out }} \cos (\theta)\right)^{\frac{3}{2}}\left(2(\alpha-\beta)^{2}+r_{2}^{2}-r_{2}^{2} \cos (2 \theta)\right)^{2}} \\
& +\frac{2 r_{2}\left((\alpha-\beta)^{2}+r_{2}^{2}-r_{2} r_{\text {in }} \cos (\theta)\right)}{\sqrt{r_{2}^{2}+r_{\text {in }}^{2}+(\alpha-\beta)^{2}-2 r_{2} r_{\text {in }} \cos (\theta)}\left(2(\alpha-\beta)^{2}+r_{2}^{2}-r_{2}^{2} \cos (2 \theta)\right)^{2}} \\
& -\frac{2 r_{2}\left((\alpha-\beta)^{2}+r_{2}^{2}-r_{2} r_{\text {out }} \cos (\theta)\right)}{\sqrt{r_{2}^{2}+r_{\text {out }}^{2}+(\alpha-\beta)^{2}-2 r_{2} r_{\text {out }} \cos (\theta)}\left(2(\alpha-\beta)^{2}+r_{2}^{2}-r_{2}^{2} \cos (2 \theta)\right)^{2}}
\end{aligned}
$$

It is noted that the expression of the axiall stiffness obtained is very simple tu use. Figure 4 represents the axial force exerted between two ring permanent magnets versus the axial displacement of the inner ring permanent magnet for the following dimensions: $r_{\text {in }}=0.025 \mathrm{~m}, r_{\text {out }}=0.028 \mathrm{~m}, r_{\text {in } 2}=0.021 \mathrm{~m}$, $r_{\text {out } 2}=0.024 \mathrm{~m}, J=1 \mathrm{~T}, h=0.003 \mathrm{~m}, z_{b}-z_{a}=0.003 \mathrm{~m}$.

\section{OPTIMIZATION OF THE INNER RING PERMANENT MAGNET DIMENSIONS}

This section discusses the optimal dimensions of the inner ring permanent magnet and the air gap in order to have either a great axial force or a great axial stiffness.

\section{A. Influence of the air gap dimension on the axial force and the axial stiffness}

First, we study the influence of the air gap width on the axial force and the axial stiffness between the two ring permanent magnets. To do so, we represent the axial force versus the axial displacement of the inner ring permanent magnet for different air gaps in Fig 5. Ii is noted that, in our configuration, the air gap width corresponds to the difference between $r_{\text {in }}$ and $r_{\text {out } 2}$. Furthermore, the width and the height of each ring permanent magnet are constant. We take $r_{\text {in }}=0.025 \mathrm{~m}, r_{\text {out }}=0.028 \mathrm{~m}, r_{\text {out } 2}-r_{\text {in } 2}=0.003 \mathrm{~m}$, 


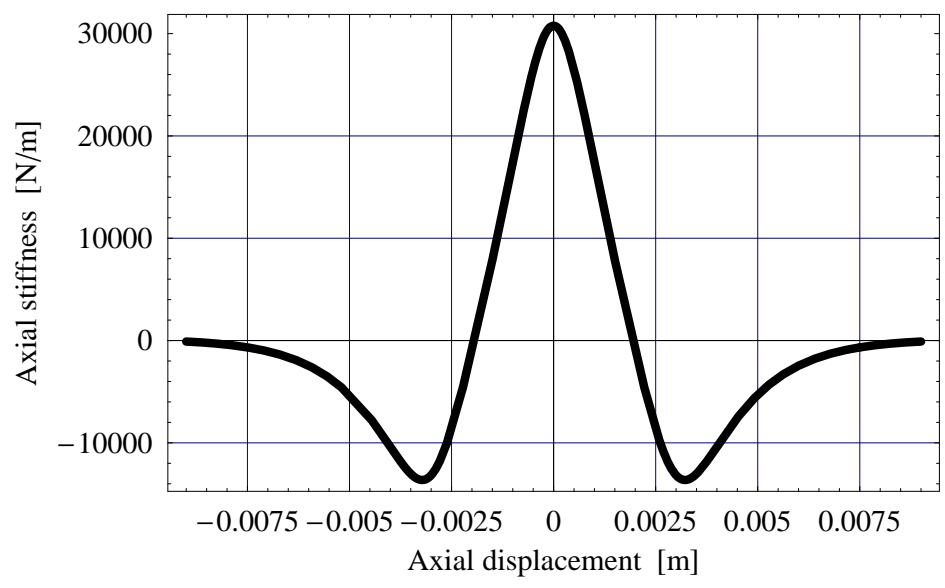

Fig. 4. Representation of the axial stiffness exerted between two ring permanent magnets axially magnetized versus the axial displacement of the inner ring permanent magnet. $r_{\text {in }}=0.025 \mathrm{~m}, r_{\text {out }}=0.028 \mathrm{~m}, r_{\text {in } 2}=0.021 \mathrm{~m}, r_{\text {out } 2}=0.024 \mathrm{~m}, J=1 \mathrm{~T}$, $h=0.003 \mathrm{~m}, z_{b}-z_{a}=0.003 \mathrm{~m}$

$J=1 \mathrm{~T}, h=0.003 \mathrm{~m}, z_{b}-z_{a}=0.003 \mathrm{~m}$.

Fig 5 shows three important points.

First, we see that the smaller the air gap between the ring permanent magnets is, the greater the axial force is. Consequently, it is necessary to have the smallest air gap between two ring permanent magnets if a great axial force is searched. This result is well-known. It was shown with the two-dimensional approach. Second, we see that the exact position of the maximal force exerted between two ring permanent magnets depends slightly on the air gap width. This result is new because our study uses a three-dimensional approach of the magnetic force whereas the previous ones used a two-dimensional approach. As a consequence, the magnet curvature must be taken into account in order to obtain precisely the position of the maximal force exerted between two ring magnets. The exact position of the maximal force versus the air gap width is represented in Fig 6. Such result is very useful because it clearly shows that if a great axial force is searched, the relative height between two rings must be chosen according to the air gap width.

Eventually, Fig 5 shows that the stiffness depends greatly on the air gap width. Indeed, we see that the smaller the air gap width is, the greater the axial stiffness is. Indeed, the gradient of the curves is the most important for small air gaps. Furthermore, this result is consistent with the representation of the axial stiffness versus the axial displacement of the inner ring permanent magnet (Fig 7). Moreover, we 


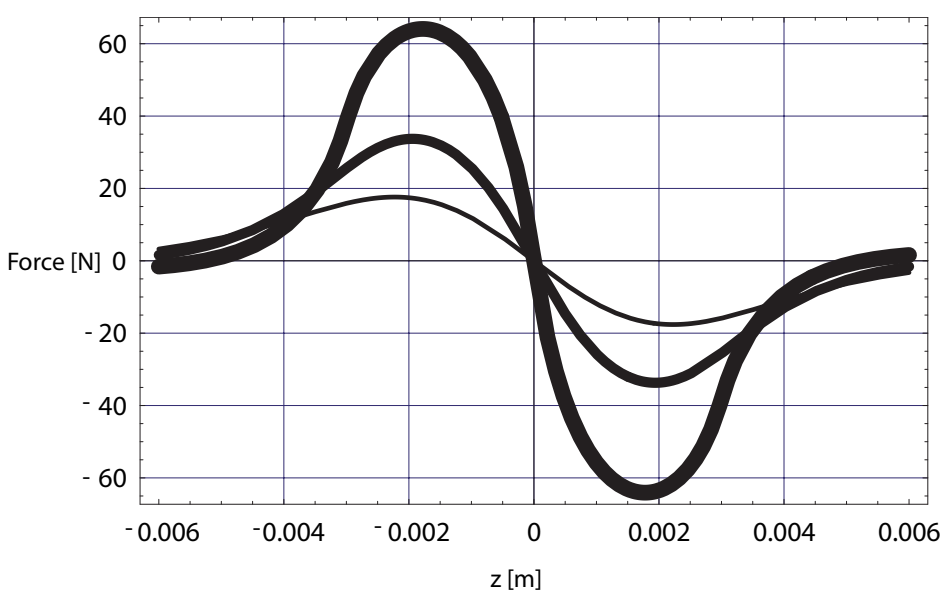

$$
\begin{aligned}
& \text { Air gap }=0.0001 \mathrm{~m} \\
& \text { Air gap }=0.001 \mathrm{~m} \\
& \text { Air gap }=0.002 \mathrm{~m}
\end{aligned}
$$

Fig. 5. Representation of the axial force exerted between two ring permanent magnets axially magnetized versus the axial displacement of the inner ring permanent magnet for different air gaps. $r_{\text {in }}=0.025 \mathrm{~m}, r_{\text {out }}=0.028 \mathrm{~m}, r_{\text {in } 2}=0.021 \mathrm{~m}$, $r_{\text {out } 2}=0.024 \mathrm{~m}, J=1 \mathrm{~T}, h=0.003 \mathrm{~m}, z_{b}-z_{a}=0.003 \mathrm{~m}$

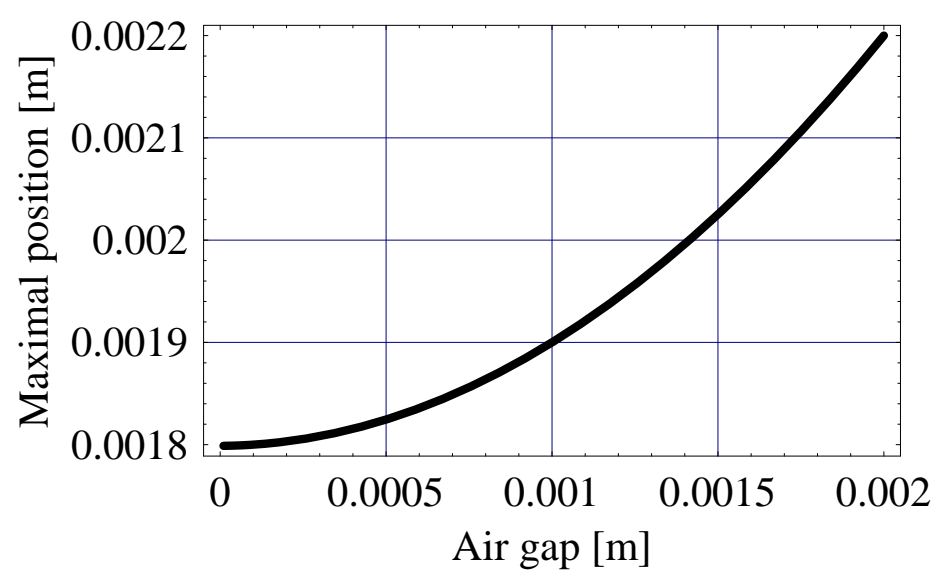

Fig. 6. Representation of the position of the maximal value of the axial force versus the air gap width: $r_{\text {in }}=0.025 \mathrm{~m}, r_{\text {out }}=$ $0.028 \mathrm{~m}, r_{\text {in } 2}=0.021 \mathrm{~m}, r_{\text {out } 2}=0.024 \mathrm{~m}, J=1 \mathrm{~T}, h=0.003 \mathrm{~m}, z_{b}-z_{a}=0.003 \mathrm{~m}$ 


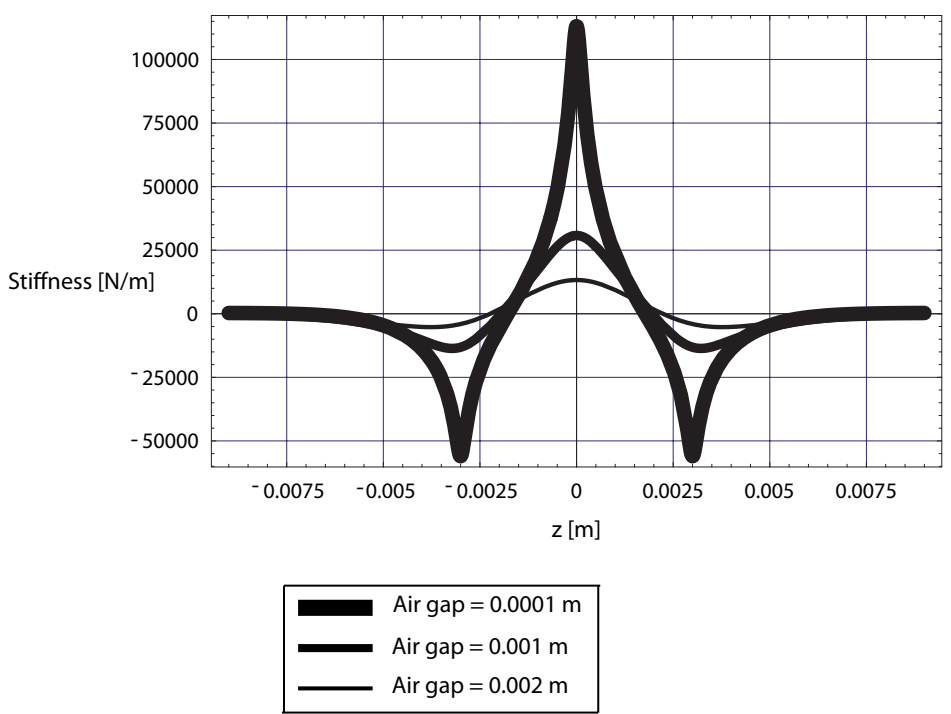

Fig. 7. Representation of the axial stiffness between two ring permanent magnets axially magnetized versus the axial displacement of the inner ring permanent magnet for different air gaps: $r_{\text {in }}=0.025 \mathrm{~m}, r_{\text {out }}=0.028 \mathrm{~m}, r_{\text {in } 2}=0.021 \mathrm{~m}, r_{\text {out } 2}=0.024 \mathrm{~m}$, $J=1 \mathrm{~T}, h=0.003 \mathrm{~m}, z_{b}-z_{a}=0.003 \mathrm{~m}$

see that when the axial force is maximal in Fig 5, the axial stiffness equals zero in Fig 7, which is still consistent.

\section{B. Determination of the optimal width of the inner ring permanent magnet}

Another parameter which can be optimized in a passive bearing is the width of the inner ring permanent magnet. To do so, the axial force is represented versus the axial displacement of the inner ring permanent magnet for several inner ring widths in Fig 8. The values taken for the parameters are the same as the previous ones. Fig 8 shows that the greater the inner ring width is, the greater the axial force is. However, it is noted that a good compromise in the ring dimensions must be found because the cost of the magnet must be taken into account. This good compromise can be found as follows: if the inner ring width equals two times its height, the axial force is $71.8 \mathrm{~N}$ when $z=-0.0018 \mathrm{~m}$. It the inner ring width equals three times its height, the axial force equals is $72.3 \mathrm{~N}$. Consequently, we deduct that it is not necessary to have an inner ring width which is greater than two times its height.

The optimal stiffness depends also on the ring inner width. To see that, we have represented in Fig 9 the axial stiffness versus the inner ring width versus the axial displacement of the inner ring permanent 


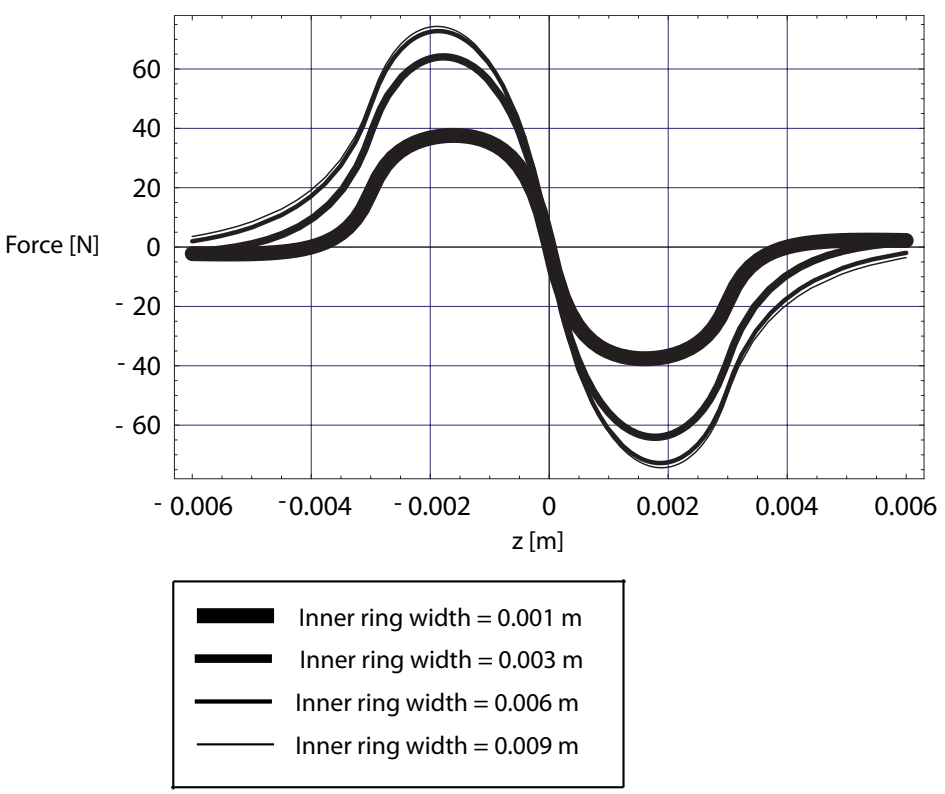

Fig. 8. Representation of the axial force between two ring permanent magnets axially magnetized versus the axial displacement of the inner ring permanent magnet for different inner ring widths: $r_{\text {in }}=0.025 \mathrm{~m}, r_{\text {out }}=0.028 \mathrm{~m}, r_{\text {in } 2}=0.021 \mathrm{~m}, r_{\text {out } 2}=0.024 \mathrm{~m}$, $J=1 \mathrm{~T}, h=0.003 \mathrm{~m}, z_{b}-z_{a}=0.003 \mathrm{~m}$

magnet. Fig 9 shows that when the inner ring width equals two times its height, a good compromise can be done for the design of magnetic passive bearings.

\section{OBTAINING THE BEST CONFIGURATION}

The previous section shows that the ring dimensions must be optimized in order to create a very good axial passive magnetic bearing. The air gap must be the smallest, the ring heights must be the same and we have shown that the inner ring width must equal two times its height. All these parameters have been determined with a outer ring whose cross-section is a square. However, we can also optimize the outer ring in order to improve the passive bearing. By taking into account the optimal dimensions found in the previous section, we can compare three configurations. The first one, shown in Fig 10-A, consists of two rings whose cross-section is a square. The second one, shown in Fig 10-B, consists of two rings whose cross-section is a rectangle whose width equals two times its height. The third one, shown in Fig 10-C, consists of two rings whose cross-section is a rectangle whose height equals two times its width. For each structure presented in Fig 10, the axial force and the axial stiffness are determined versus the 


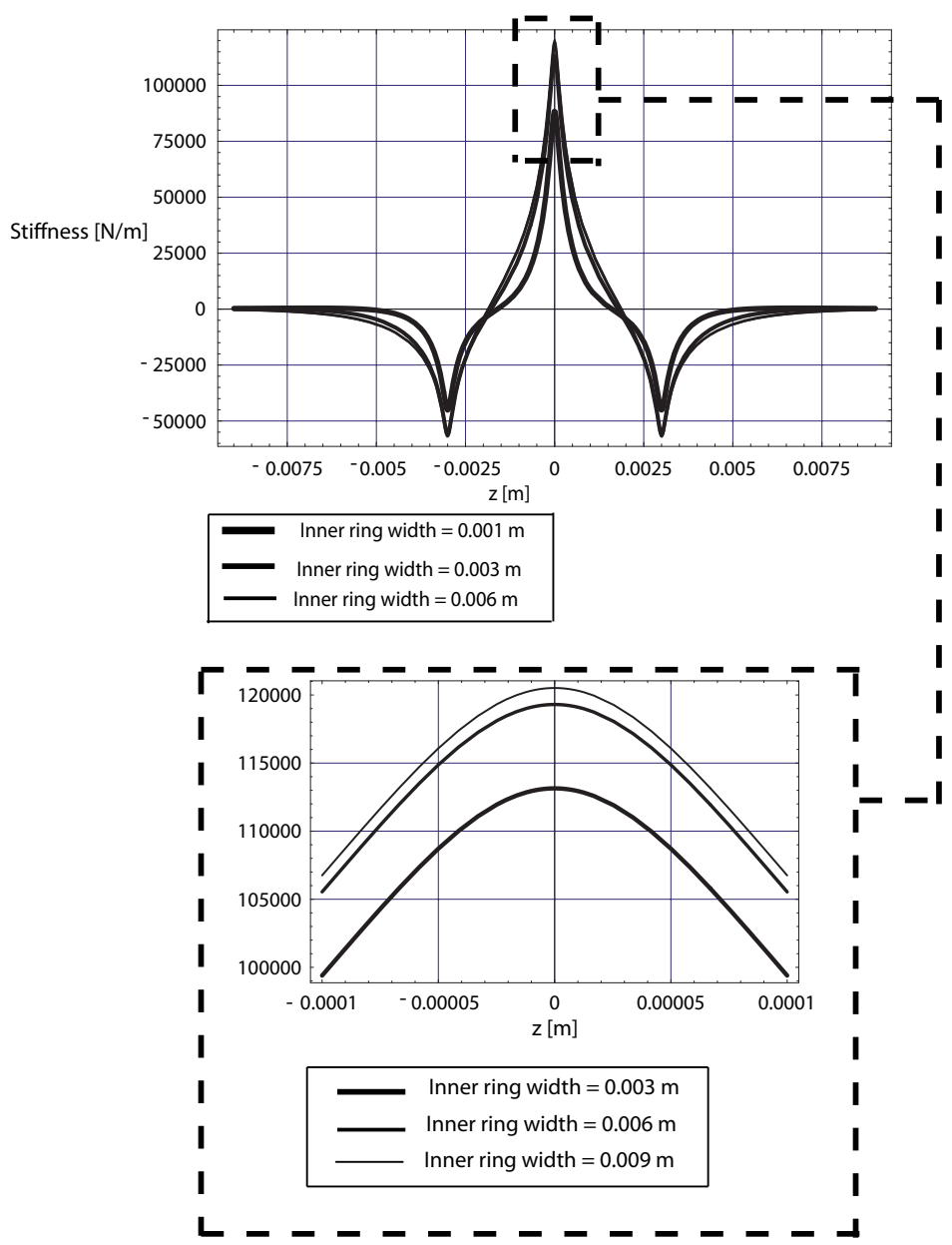

Fig. 9. Representation of the axial stiffness between two ring permanent magnets axially magnetized versus the axial displacement of the inner ring permanent magnet for different inner ring widths: $r_{\text {in }}=0.025 \mathrm{~m}, r_{\text {out }}=0.028 \mathrm{~m}, r_{\text {in } 2}=0.021 \mathrm{~m}, r_{\text {out } 2}=0.024 \mathrm{~m}$, $J=1 \mathrm{~T}, h=0.003 \mathrm{~m}, z_{b}-z_{a}=0.003 \mathrm{~m}$

axial displacement of the inner ring permanent magnet. The axial force is shown in Fig 11 and the axial stiffness is shown in Fig 12.

Fig 11 shows that the best configuration is order to have a great axial force is the configuration presented in Fig 10-B. Fig 12 shows that two configurations are equivalent for the case when the greatest axial stiffness is searched. Consequently, according to the involved application, the configurations presented in Fig 10-B and 10-C can be used.

At least, we can say that the configuration presented in Fig 10-A is the least interesting configuration. 


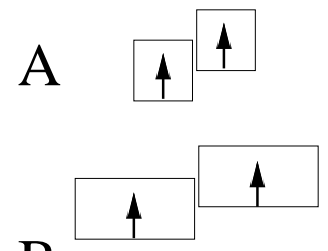

$\mathrm{B}$

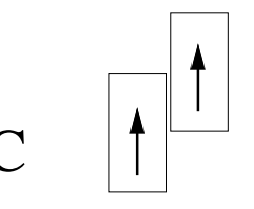

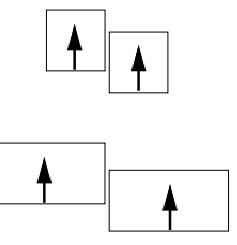

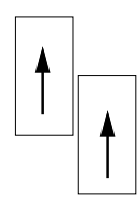

Fig. 10. Representation of three passive magnetic bearings. $\mathrm{A}=r_{\text {in }}=0.025 \mathrm{~m}, r_{\text {out }}=0.028 \mathrm{~m}, r_{\text {out } 2}=0.0249 \mathrm{~m}, r_{\text {in } 2}=$ $0.0219 \mathrm{~m}, h=0.003 \mathrm{~m}, z b-z_{a}=0.003 \mathrm{~m}$. B $=r_{\text {in }}=0.025 \mathrm{~m}, r_{\text {out }}=0.031 \mathrm{~m}, r_{\text {out } 2}=0.0249 \mathrm{~m}, r_{\text {in } 2}=0.0189 \mathrm{~m}$, $h=0.003 \mathrm{~m}, z b-z_{a}=0.003 \mathrm{~m} . \mathrm{C}=r_{\text {in }}=0.025 \mathrm{~m}, r_{\text {out }}=0.028 \mathrm{~m}, r_{\text {out } 2}=0.0249 \mathrm{~m}, r_{\text {in } 2}=0.0219 \mathrm{~m}, h=0.006 \mathrm{~m}$, $z b-z_{a}=0.006 \mathrm{~m}$.
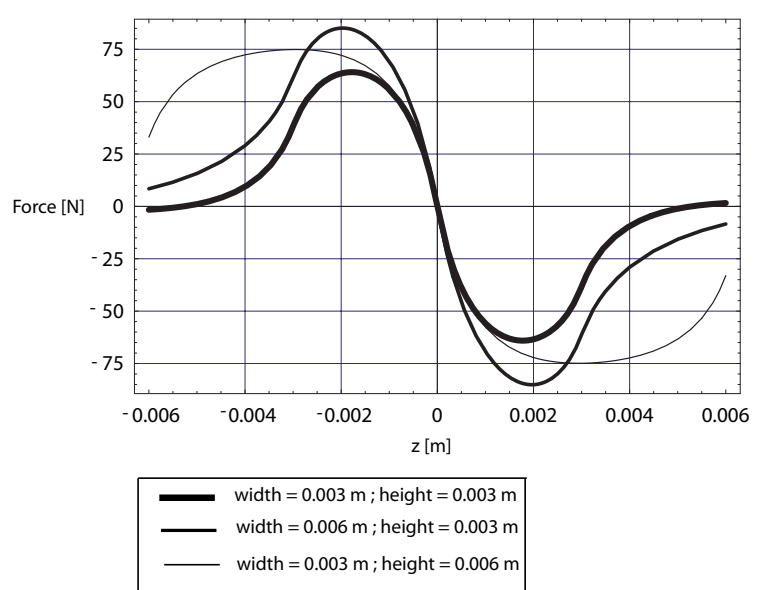

Fig. 11. Representation of the axial force exerted between two ring permanent magnets versus inner ring width $r_{i n}=0.025 \mathrm{~m}$, $r_{\text {out }}=0.028 \mathrm{~m}, r_{\text {out } 2}=0.0249 \mathrm{~m}, J=1 \mathrm{~T}, h=0.003 \mathrm{~m}, z b-z_{a}=0.003 \mathrm{~m}$. 


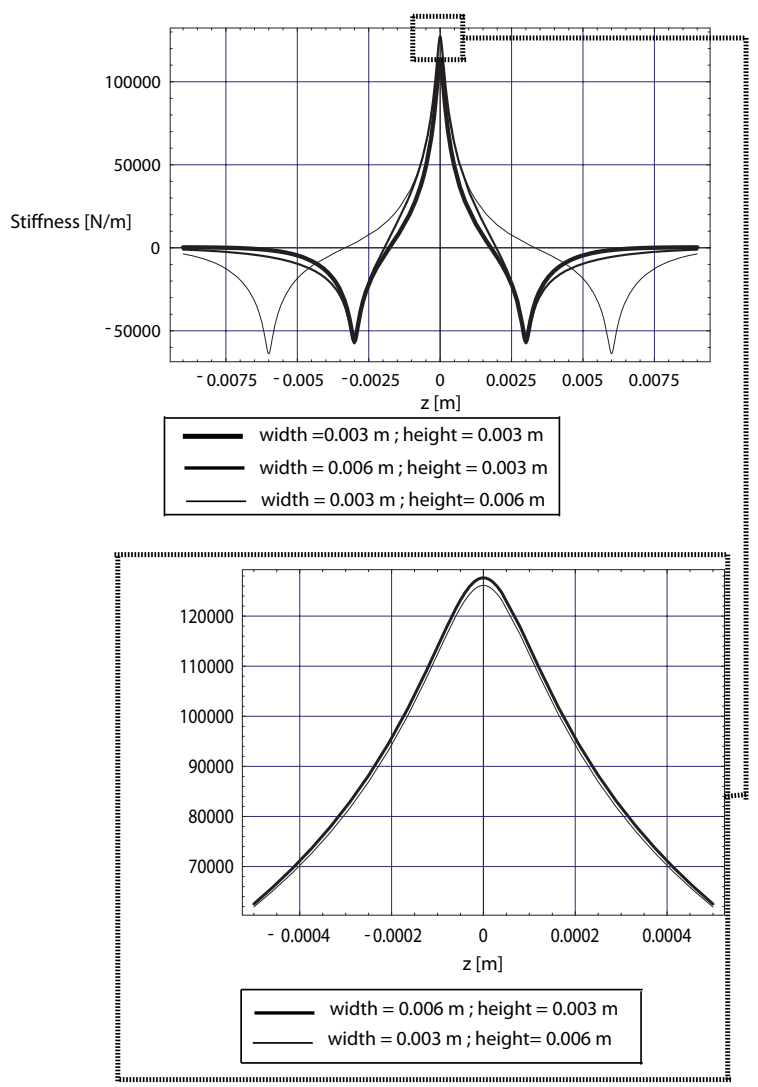

Fig. 12. Representation of the axial stiffness exerted between two ring permanent magnets versus inner ring width $r_{i n}=0.025 \mathrm{~m}$, $r_{\text {out }}=0.028 \mathrm{~m}, r_{\text {out } 2}=0.0249 \mathrm{~m}, J=1 \mathrm{~T}, h=0.003 \mathrm{~m}, z b-z_{a}=0.003 \mathrm{~m}$.

\section{CONCLUSION}

This paper has presented the semi-analytical expressions of the force and the stiffness exerted between two ring permanent magnets whose polarization is axial. Such a configuration corresponds to an axial passive magnetic bearing. Thanks to the exact expressions of the force and the stiffness, we have shown that we can optimize the ring dimensions in order to have either a great force or a great stiffness. A great force can be obtained if the ring width equals two times its height. Then, we have shown that two configurations are equivalent for the case when a great axial stiffness is searched.

\section{REFERENCES}

[1] J. P. Yonnet, "Passive magnetic bearings with permanent magnets," IEEE Trans. Magn., vol. 14, no. 5, pp. 803-805, 1978. 
[2] J. P. Yonnet, "Permanent magnet bearings and couplings," IEEE Trans. Magn., vol. 17, no. 1, pp. 1169-1173, 1981.

[3] B. Azzerboni, E. Cardelli, and A. Tellini, "Computation of the magnetic field in massive conductor systems," IEEE Trans. on Magn., vol. 25, no. 6, pp. 4462-4473, 1989.

[4] E. P. Furlani, S. Reznik, and A. Kroll, "A three-dimensonal field solution for radially polarized cylinders," IEEE Trans. Magn., vol. 31, no. 1, pp. 844-851, 1995.

[5] G. Akoun and J. P. Yonnet, "3d analytical calculation of the forces exerted between two cuboidal magnets," IEEE Trans. Magn., vol. 20, no. 5, pp. 1962-1964, 1984.

[6] K. Kim, E. Levi, Z. Zabar, and L. Birenbaum, "Mutual inductance of noncoaxial circular coils with constant current density," IEEE Trans. Magn., vol. 33, no. 32, pp. 4303-4309, 1997.

[7] J. P. Selvaggi, S. Salon, O. M. Kwon, and M. V. K. Chari, "Calculating the external magnetic field from permanent magnets in permanent-magnet motors - an alternative method," IEEE Trans. Magn., vol. 40, no. 5, pp. 3278-3285, 2004.

[8] J. Conway, "Inductance calculations for noncoaxial coils using bessel functions," IEEE Trans. Magn., vol. 43, no. 3, pp. 10231034, 2007.

[9] J. Conway, "Noncoaxial inductance calculations without the vector potential for axisymmetric coils and planar coils," IEEE Trans. Magn., vol. 44, no. 10, pp. 453-462, 2008.

[10] J. P. Selvaggi, S. Salon, O. M. Kwon, and M. V. K. Chari, "Computation of the three-dimensional magnetic field from solid permanent-magnet bipolar cylinders by employing toroidal harmonics," IEEE Trans. Magn., vol. 43, no. 10, pp. 3833-3839, 2007.

[11] K. Kim, E. Levi, Z. Zabar, and L. Birenbaum, "Restoring force between two noncoaxial circular coils," IEEE Trans. Magn., vol. 32, no. 32, pp. 478-484, 1996.

[12] S. Babic, C. Akyel, S. Salon, and S. Kincic, "New expressions for calculating the magnetic field created by radial current in massive disks," IEEE Trans. Magn., vol. 38, no. 2, pp. 497-500, 2002.

[13] S. Babic and M. M. Gavrilovic, "New expression for calculating magnetic fields due to current-carrying solid conductors," IEEE Trans. Magn., vol. 33, no. 5, pp. 4134-4136, 1997.

[14] P. Samanta and H. Hirani, "Magnetic bearing configurations: Theoretical and experimental studies," IEEE Trans. Magn., vol. 44, no. 2, pp. 292-300, 2008.

[15] B. Azzerboni and G. Saraceno, "Three-dimensional calculation of the magnetic field created by current-carrying massive disks," IEEE Trans. Magn., vol. 34, no. 5, pp. 2601-2604, 1998.

[16] B. Azzerboni and E. Cardelli, "Magnetic field evaluation for disk conductors," IEEE Trans. Magn., vol. 29, no. 6, pp. 24192421, 1993.

[17] B. Azzerboni, E. Cardelli, M. Raugi, A. Tellini, and G. Tina, "Analytic expressions for magnetic field from finite curved conductors," IEEE Trans. Magn., vol. 27, no. 2, pp. 750-757, 1991.

[18] C. Blache and G. Lemarquand, "New structures for linear displacement sensor with hight magnetic field gradient," IEEE Trans. Magn., vol. 28, no. 5, pp. 2196-2198, 1992.

[19] G. Lemarquand and V. Lemarquand, “Annular magnet position sensor," IEEE. Trans. Magn., vol. 26, no. 5, pp. 2041-2043, 1990.

[20] H. L. Rakotoarison, J. P. Yonnet, and B. Delinchant, "Using coulombian approach for modeling scalar potential and magnetic field of a permanent magnet with radial polarization," IEEE Trans. Magn., vol. 43, no. 4, pp. 1261-1264, 2007.

[21] V. Lemarquand, J. F. Charpentier, and G. Lemarquand, "Nonsinusoidal torque of permanent-magnet couplings," IEEE Trans. Magn., vol. 35, no. 5, pp. 4200-4205, 1999. 
[22] J. F. Charpentier and G. Lemarquand, "Calculation of ironless permanent magnet coupling using semi-numerical magnetic pole theory method," COMPEL, vol. 20, no. 1, pp. 72-89, 2001.

[23] P. Elies and G. Lemarquand, "Analytical optimization of the torque of a permanent-magnet coaxial synchronous coupling," IEEE Trans. Magn., vol. 34, no. 4, pp. 2267-2273, 1998.

[24] Z. Zhu, Z. Xia, and D. Howe, "Comparison of halbach magnetized brushless machines based on discrete magnet segments or a single ring magnet," IEEE Trans. Magn., vol. 38, no. 9, pp. 2997-2999, 2002.

[25] R. Ravaud, G. Lemarquand, V. Lemarquand, and C. Depollier, "Analytical calculation of the magnetic field created by permanent-magnet rings," IEEE Trans. Magn., vol. 44, no. 8, pp. 1982-1989, 2008.

[26] R. Ravaud, G. Lemarquand, V. Lemarquand, and C. Depollier, "The three exact components of the magnetic field created by a radially magnetized tile permanent magnet.," Progress in Electromagnetics Research, PIER 88, pp. 307-319, 2008.

[27] R. Ravaud, G. Lemarquand, V. Lemarquand, and C. Depollier, "Discussion about the analytical calculation of the magnetic field created by permanent magnets.," Progress in Electromagnetics Research B, vol. 11, pp. 281-297, 2009.

[28] R. Ravaud, G. Lemarquand, V. Lemarquand, and C. Depollier, "Magnetic field produced by a tile permanent magnet whose polarization is both uniform and tangential," Progress in Electromagnetics Research B, vol. 13, pp. 1-20, 2009.

[29] E. Durand, Magnetostatique. Masson Editeur, Paris, France, 1968.

[30] S. Babic and C. Akyel, "Improvement in the analytical calculation of the magnetic field produced by permanent magnet rings," Progress in Electromagnetics Research C, vol. 5, pp. 71-82, 2008.

[31] http://www.univ-lemans.fr/ glemar. 